\title{
Achieving sustainable transport through resource scheduling: A case study for electric vehicle charging stations
}

\author{
Gong, D. ${ }^{a}$, Tang, M. ${ }^{\text {a, }}{ }^{,}$, Liu, S. ${ }^{a}$, Xue, G. ${ }^{a}$, Wang, L. ${ }^{a}$ \\ ${ }^{\mathrm{a}}$ School of Economics and Management, Beijing Jiaotong University, Beijing, P.R. China
}

\begin{abstract}
A B S T R A C T
Electric vehicles support low-carbon emissions to revitalize sustainable transportation, and more charging stations are being built to meet the daily charging demand. Charging piles and service workers are the most important resources for electric vehicle charging stations, and the scheduling of these resources is an important factor affecting the charging stations' profits and sustainable industrial development. In this paper, we simulate the charging piles and service workers in charging station resource scheduling and analyze the impacts of the number of service workers, the charging pile replacement policy and the charging pile maintenance times on an electric vehicle charging station's profits. An orthogonal test can achieve the following optimal resource scheduling results when their range is known: (1) In the lifetime of the charging pile, seven maintenance times are needed; (2) Even if the charging pile is still in normal condition, it needs to be replaced in order to achieve the maximum profits for the charging station; (3) a comprehensive analysis of service efficiency and service costs indicates that 8 service workers are needed to achieve the optimal profits for the charging station. Therefore, the scientific contribution of this research is to establish one resource scheduling simulation model that can assess the effects of the number of service workers, the charging pile replacement policy and the charging pile maintenance times on charging station revenues and to obtain the optimal results. In addition, if the model parameters change, we can still obtain the optimal results.
\end{abstract}

\section{ARTICLE INFO}

Keywords:

Sustainable transport;

Resource scheduling;

Electric vehicle;

Charging station;

Simulation;

Profit

*Corresponding author:

mincong@bjtu.edu.cn

(Tang, M.)

Article history:

Received 8 September 2018

Revised 12 February 2019

Accepted 24 February 2019

\section{References}

[1] Liu, S., Gong, D. (2014). Modelling and simulation on recycling of electric vehicle batteries - Using agent approach, International Journal of Simulation Modelling, Vol. 13, No. 1, 79-92, doi: 10.2507/IJSIMM13(1)CO1.

[2] Johanyák, Z.C. (2017). A modified particle swarm optimization algorithm for the optimization of a fuzzy classification subsystem in a series hybrid electric vehicle, Tehnički Vjesnik - Technical Gazette, Vol. 24, Supplement 2, 295-301, doi: 10.17559/TV-20151021202802.

[3] Webster, R. (1999). Can the electricity distribution network cope with an influx of electric vehicles?, Journal of Power Sources, Vol. 80, No. 1-2, 217-225, doi: 10.1016/S0378-7753(98)00262-6.

[4] Tamor, M.A., Gearhart, C., Soto, C. (2013). A statistical approach to estimating acceptance of electric vehicles and electrification of personal transportation, Transportation Research Part C: Emerging Technologies, Vol. 26, 125134, doi: 10.1016/j.trc.2012.07.007.

[5] Nie, Y.(M.), Ghamami, M. (2013). A corridor-centric approach to planning electric vehicle charging infrastructure, Transportation Research Part B: Methodological, Vol. 57, 172-190, doi: 10.1016/j.trb.2013.08.010.

[6] Lu, X.C., Chen, Q.B., Zhang, Z.J. (2014). The electric vehicle routing optimizing algorithm and the charging stations' layout analysis in Beijing, International Journal of Simulation Modelling, Vol. 13, No. 1, 116-127, doi: $10.2507 /$ IJSIMM13(1)CO4.

[7] Simeunović, N., Kamenko, I., Bugarski, V., Jovanović, M., Lalić, B. (2017). Improving workforce scheduling using artificial neural networks model, Advances in Production Engineering \& Management, Vol. 12, No. 4, 337-352, doi: 
10.14743/apem2017.4.262.

[8] Miller, R.E., Thatcher, J.W. Bohlinger, J.D. (eds.), (1972). Complexity of computer computations, Springer Verlag, Boston, USA, doi: 10.1007/978-1-4684-2001-2.

[9] Cook, S.A. (1971). The complexity of theorem-proving procedures, In: Proceedings of the third annual ACM symposium on theory of computing STOC '71, Ohio, USA, 151-158, doi: 10.1145/800157.805047.

[10] Xi, X., Sioshansi, R., Marano, V. (2013). Simulation-optimization model for location of a public electric vehicle charging infrastructure, Transportation Research Part D: Transport and Environment, Vol. 22, 60-69, doi: 10.1016 /j.trd.2013.02.014.

[11] Zhang, L., Shaffer, B., Brown, T., Samuelsen, G.S. (2015). The optimization of DC fast charging deployment in California, Applied Energy, Vol. 157, 111-122, doi: 10.1016/j.apenergy.2015.07.057.

[12] Chen, T.D., Kockelman, K.M., Khan, M. (2013). Locating electric vehicle charging stations: Parking-based assignment method for Seattle, Washington, Transportation Research Record: Journal of the Transportation Research Board, Vol. 2385, No. 1, 28-36, doi: 10.3141/2385-04.

[13] Yi, Z., Bauer, P.H. (2016). Optimization models for placement of an energy-aware electric vehicle charging infrastructure, Transportation Research Part E: Logistics and Transportation Review, Vol. 91, 227-244, doi: 10.1016/ j.tre.2016.04.013.

[14] Mehar, S., Senouci, S.M. (2013). An optimization location scheme for electric charging stations, In: Proceedings of International Conference on Smart Communications in Network Technologies (SaCoNeT) 2013, Paris, France, 1-5, doi: 10.1109/SaCoNeT.2013.6654565.

[15] Bendiabdellah, Z., Senouci, S.M., Feham, M. (2014). A hybrid algorithm for planning public charging stations, In: Global Information Infrastructure and Networking Symposium (GIIS) 2014, Montreal, Canada, 1-3, doi: 10.1109/ GIIS.2014.6934262.

[16] You, P.-S., Hsieh, Y.-C. (2014). A hybrid heuristic approach to the problem of the location of vehicle charging stations, Computers \& Industrial Engineering, Vol. 70, 195-204, doi: 10.1016/i.cie.2014.02.001.

[17] Tang, M., Gong, D., Liu, S., Zhang, H. (2016). Applying multi-phase particle swarm optimization to solve bulk cargo port scheduling problem, Advances in Production Engineering \& Management, Vol. 11, No. 4, 299-310, doi: 10.14743/apem2016.4.228

[18] Guo, S., Zhao, H. (2015). Optimal site selection of electric vehicle charging station by using fuzzy TOPSIS based on sustainability perspective, Applied Energy, Vol. 158, 390-402, doi: 10.1016/j.apenergy.2015.08.082.

[19] Wu, Y., Chen, K., Zeng, B., Yang, M., Geng, S. (2016). Cloud-based decision framework for waste-to-energy plant site selection - A case study from China, Waste Management, Vol. 48, 593-603, doi: 10.1016/j.wasman.2015. $\underline{11.030}$.

[20] Shafiei, E., Thorkelsson, H., Ásgeirsson, E.I., Davidsdottir, B., Raberto, M., Stefansson, H. (2012). An agent-based modeling approach to predict the evolution of market share of electric vehicles: A case study from Iceland, Technological Forecasting and Social Change, Vol. 79, No. 9, 1638-1653, doi: 10.1016/i.techfore.2012.05.011.

[21] Adepetu, A., Keshav, S., Arya, V. (2016). An agent-based electric vehicle ecosystem model: San Francisco case study, Transport Policy, Vol. 46, 109-122, doi: 10.1016/j.tranpol.2015.11.012.

[22] Ghamami, M., Nie, Y.(M.), Zockaie, A. (2016). Planning charging infrastructure for plug-in electric vehicles in city centers, International Journal of Sustainable Transportation, Vol. 10, No. 4, 343-353, doi: 10.1080/15568318. 2014.937840

[23] Yıldız, B., Arslan, O., Karaşan, O.E. (2016). A branch and price approach for routing and refueling station location model, European Journal of Operational Research, Vol. 248, No. 3, 815-826, doi: 10.1016/i.ejor.2015.05.021.

[24] Cavadas, J., Correia, G.H.D.A., Gouveia, J. (2015). A MIP model for locating slow-charging stations for electric vehicles in urban areas accounting for driver tours, Transportation Research Part E: Logistics and Transportation Review, Vol. 75,188-201, doi: 10.1016/j.tre.2014.11.005.

[25] Dorling, K., Heinrichs, J., Messier, G.G., Magierowski, S. (2017). Vehicle routing problems for drone delivery, IEEE Transactions on Systems, Man, and Cybernetics: Systems, Vol. 47, No. 1, 70-85, doi: 10.1109/TSMC.2016.2582745.

[26] Tang, M., Gong, D., Liu, S., Lu, X. (2017). Finding key factors affecting the locations of electric vehicle charging stations: A simulation and ANOVA approach, International Journal of Simulation Modelling, Vol. 16, No. 3, 541554, doi: 10.2507/IJSIMM16(3)C015.

[27] Manley, M., Kim, Y.S., Christensen, K., Chen, A. (2016). Airport emergency evacuation planning: An agent-based simulation study of dirty bomb scenarios, IEEE Transactions on Systems, Man, and Cybernetics: Systems, Vol. 46, No. 10, 1390-1403, doi: 10.1109/TSMC.2015.2497213. 
APEM
Advances in Production Engineering \& Management Letnik 14 | Številka 1 | Marec 2019 | Strani 65-79 https://doi.org/10.14743/apem2019.1.312
ISSN 1854-6250

Spletna stran: apem-journal.org Izvirni znanstveni članek

\title{
Doseganje trajnostnega prometa $\mathrm{z}$ razporejanjem virov: Študija primera za polnilne postaje za električna vozila
}

\author{
Gong, D. ${ }^{a}$, Tang, M. ${ }^{a,}{ }^{*}$, Liu, S. ${ }^{a}$, Xue, G. ${ }^{a}$, Wang, L. ${ }^{a}$ \\ ${ }^{\mathrm{a}}$ School of Economics and Management, Beijing Jiaotong University, Beijing, P.R. China
}

\section{POVZETEK}

Električna vozila podpirajo nizkoogljične emisije za oživitev trajnostnega prometa, za zadovoljevanje dnevnih potreb po polnjenju pa se gradi več polnilnih postaj. Polnilna mesta in servisni delavci so najpomembnejši viri polnilnih postaj za električna vozila, razporeditev teh virov pa je pomemben dejavnik, ki vpliva na dobiček polnilnih postaj in trajnostni industrijski razvoj. $\mathrm{V}$ tem prispevku simuliramo razporejanje virov polnilnih postaj in analiziramo vpliv števila servisnih delavcev, pravilnika o zamenjavi polnilnih mest in vzdrževalnih ciklov polnilnih mest na dobiček polnilne postaje. Pravokotni eksperiment lahko doseže naslednje optimalne rezultate razporejanja virov, ko je njihov obseg znan: (1) v življenjski dobi polnilnega mesta je potrebnih sedem vzdrževalnih ciklov; (2) tudi če je polnilno mesto še vedno v normalnem stanju, ga je treba zamenjati, da dosežemo največji dobiček polnilne postaje; (3) celovita analiza učinkovitosti storitev in stroškov storitev kaže, da je za doseganje optimalnih dobičkov za polnilno postajo potrebnih osem uslužbencev. Zato je znanstveni prispevek te raziskave vzpostavitev enotnega simulacijskega modela razporejanja virov, ki lahko oceni učinke števila uslužbencev, politike zamenjave polnilnih mest in vzdrževalnih ciklov polnilnih mest na prihodke polnilnih postaj in doseže optimalne rezultate. Poleg tega lahko dobimo optimalne rezultate tudi, če se parametri modela spremenijo.

\section{PODATKI O ČLANKU}

Ključne besede:

Trajnostni promet;

Razpored virov;

Električno vozilo;

Polnilna postaja;

Simulacija;

Dobiček

*Kontaktna oseba:

mincong@bjtu.edu.cn

(Tang, M.)

Zgodovina članka:

Prejet 8. septembra 2018

Popravljen 12. februarja 2019

Sprejet 24. februarja 2019 